Danezza Mae D. Lim, MD

Nathaniel W. Yang, MD

Department of Otorhinolaryngology

Head and Neck Surgery

The Medical City

\section{Meningioma in the Middle Ear: An Unusual Case of Hearing Loss}

When evaluating patients presenting with progressive unilateral hearing loss without a history of trauma or infection, it is important consider the clinical correlation of physical examination findings, imaging and audiograms. It is crucial that all findings are correctly reviewed and analyzed to provide an accurate assessment and appropriate management for the patient.

\section{CASE REPORT}

A 46-year-old woman presented with a gradually progressing left-sided hearing loss with associated continuous tinnitus for a period of 14 months. There was no history of trauma or ear infection. On prior consultation with an ENT specialist, the patient was diagnosed to have otitis media with effusion and initially managed medically.

Persistence of symptoms without improvement prompted a follow-up consult where pure tone audiometry (PTA) and speech testing showed normal hearing in the right ear, and a moderate to severe mixed hearing loss in the left ear. (Figure 1) Significant bleeding was encountered on myringotomy, and the possibility of a glomus tympanicum was entertained.

A plain temporal bone CT Scan revealed a soft tissue density occupying the left middle ear space, with bony erosion in the area of the jugular foramen which suggested the possibility of a tympanojugular paraganglioma. (Figure 2) The patient was subsequently referred to our institution for surgical management.

On physical examination, the left tympanic membrane was bulging with note of a pinkish retrotympanic mass. Lateralization to the right was noted on Weber testing using $0.5,1$ and $2 \mathrm{kHz}$ tuning forks. Subjectively, the patient could not hear anything on the left side. Due to the inconsistency noted between the tuning fork test finding and the initial audiometric test result, the patient was advised to undergo a second audiometric test under the supervision of a reliable audiologist. This second audiogram revealed normal hearing in the right and profound hearing loss in the left ear (Figure 3), a finding that was consistent with the patient's subjective hearing perception and tuning fork test results. 


\section{FEATURED GRAND ROUNDS}

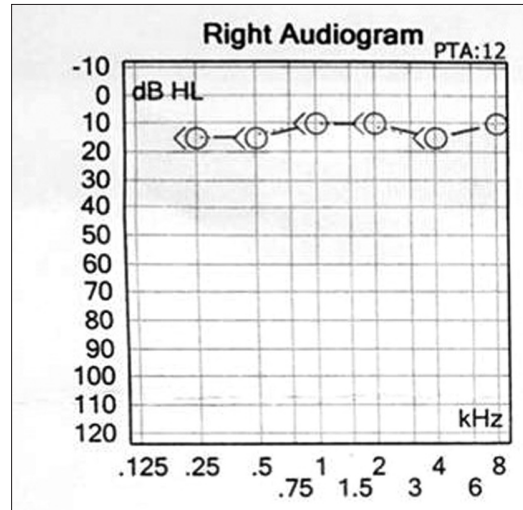

Effective masking for test ear right

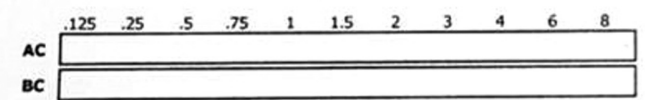

PURE TONE AUDIOGRAM

\begin{tabular}{|c|c|c|}
\hline sight & Ky & Let? \\
\hline 0 & $A C$ & $x$ \\
\hline$\Delta$ & Mateose & ㅁ \\
\hline$<$ & cone & $>$ \\
\hline [ & 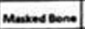 & \\
\hline$M$ & мас & $M$ \\
\hline U & vat & U \\
\hline s & sones. & $s$ \\
\hline 8 & Acenestat & $B$ \\
\hline 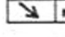 & 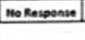 & \\
\hline
\end{tabular}

Degree of Hearing Loss (WHO)

0.25 dB - Norma

41-55 $\mathrm{dB}$. Moderate

56.70 dB - Moderate to Severe

$71 \cdot 90 \mathrm{~dB}$ - Severe

$91 \mathrm{~dB}$ and above - Protoun

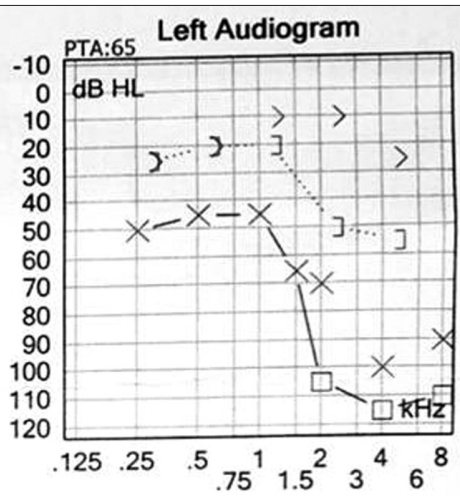

Effective masking for test ear left

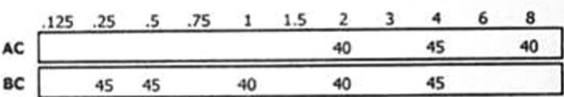

SPEECH AUDIOMETRY

SRT PTA Right: 11.7 PTA Left: 65.0 Transducer Test type Intensity Masking Aided ISF440 List Transducer

Left $\mathrm{HL}$

$\mathrm{HL}$ 15

70
N/A

Right

WR

WR PTA Right: 11.7 PTA Left: 65.0 Intensity Masking Score Aided ISF440 List

Figure 1. Audiogram, Right: Normal Hearing; Left: Downsloping moderate to severe mixed hearing loss. Note that masking was not done consistently on the left, and that the speech discrimination score of 80 on the left was significantly lower than the score of 96 on the right.
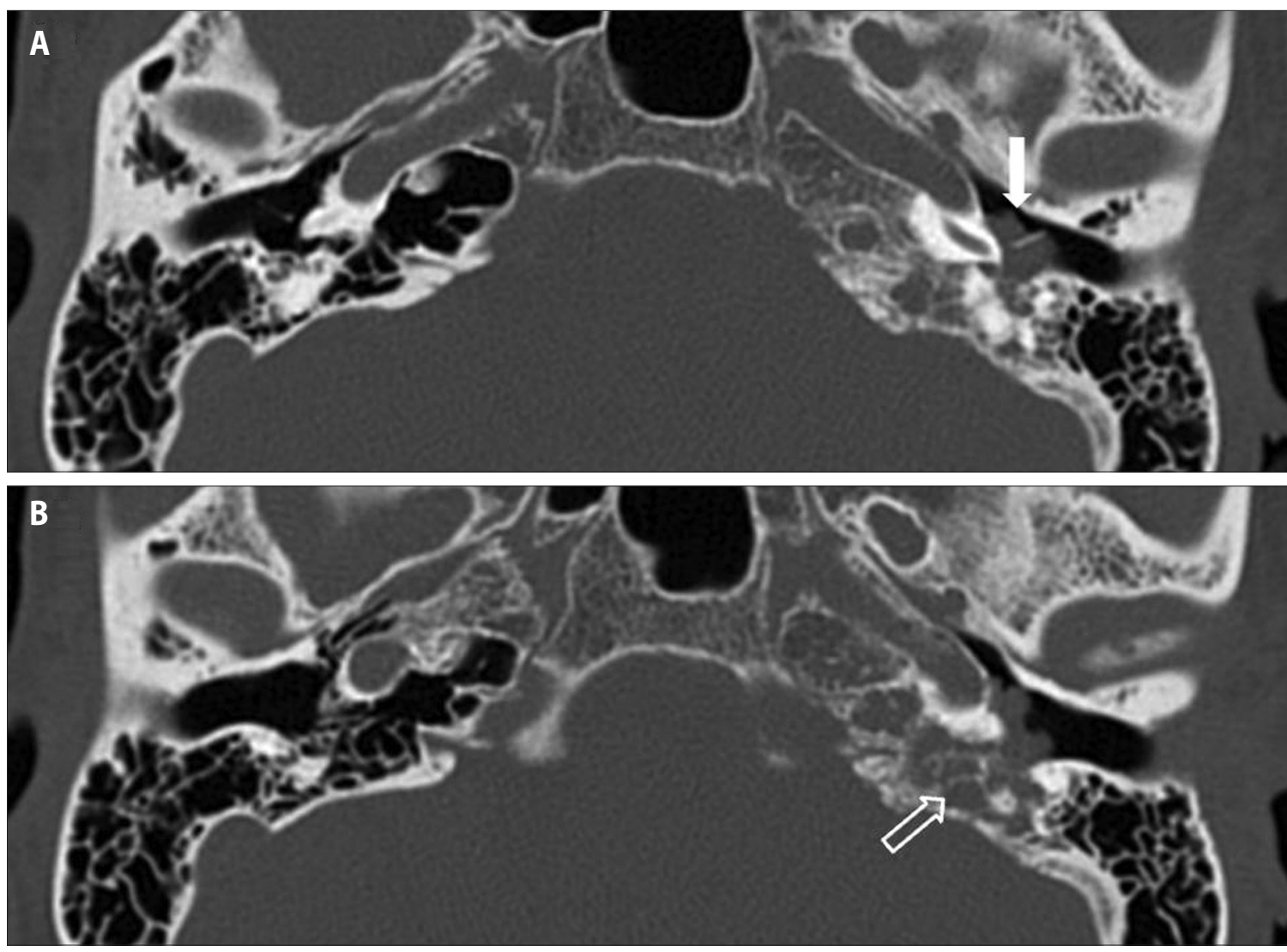

Figure 2. CT of the temporal bone, axial views. Figure $\mathbf{A}$. Soft tissue lesion occupying a portion of the middle ear space at the level of the basal turn of the cochlea (solid white arrow). B. shows soft tissue infiltration in the region of the jugular foramen with patchy bone destruction (outlined white arrow). 


\section{FEATURED GRAND ROUNDS}

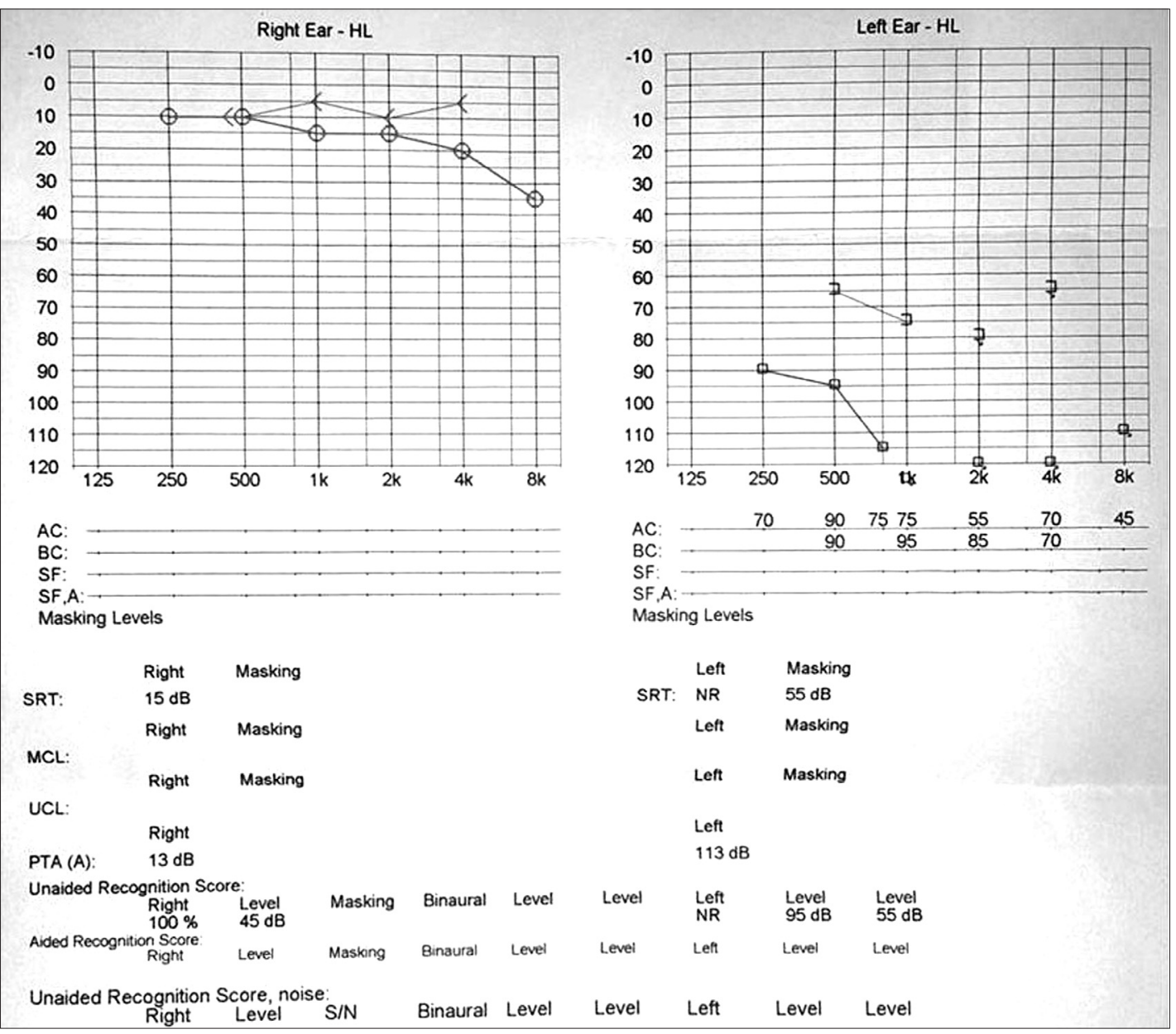

Figure 3. Repeat Audiogram. Right: Normal hearing threshold sloping to mild hearing loss; Left: Profound hearing loss, SRT conforms with the PTA, SDS $100 \%$ on the right, no response on the left.

With the finding of a sensorineural rather than a conductive type of hearing loss, additional imaging to investigate the presence of retrocochlear pathology became necessary. A Gd-enhanced MRI examination revealed the presence of a $2.6 \times 4.5 \mathrm{~cm}$ cerebello-pontine angle mass causing pressure on the adjacent pons and left cerebellar hemisphere. The lesion appeared to arise from the posterior face of the temporal bone, with extension into the jugular foramen and middle ear (Figure 4). A dural tail sign was likewise noted, and this led to a change in diagnosis to a posterior fossa meningioma with extension into the temporal bone and middle ear.

The patient was then referred to a neurosurgeon, who expressed some doubt regarding the diagnosis and requested a biopsy. Due to the accessibility of the middle ear component, the patient underwent a transcanal middle ear exploration with biopsy. The histopathology report confirmed the impression of meningioma (meningotheliomatous subtype).The patient subsequently underwent neurosurgical management for the intracranial portion of the tumor.

\section{DISCUSSION}

Neoplasms of the cerebellopontine angle (CPA) are most common in the posterior cranial fossa which account for $5-10 \%$ of all intracranial tumors. ${ }^{1}$ The two most common lesions which comprise $79 \%$ of incidents recorded are vestibular schwannomas and meningiomas. Other pathologies include epidermoid cysts, facial and lower cranial nerve schwannomas, and arachnoid cysts. ${ }^{2}$

Up to $20 \%$ of intracranial meningiomas may have extracranial extension. ${ }^{3-5}$ These areas may include the scalp, sinonasal tract, orbit, soft tissues, ear and temporal bone. Due to its low invasive properties, incidence of extension particularly to the middle ear is less than $2 \%{ }^{4-6}$ 


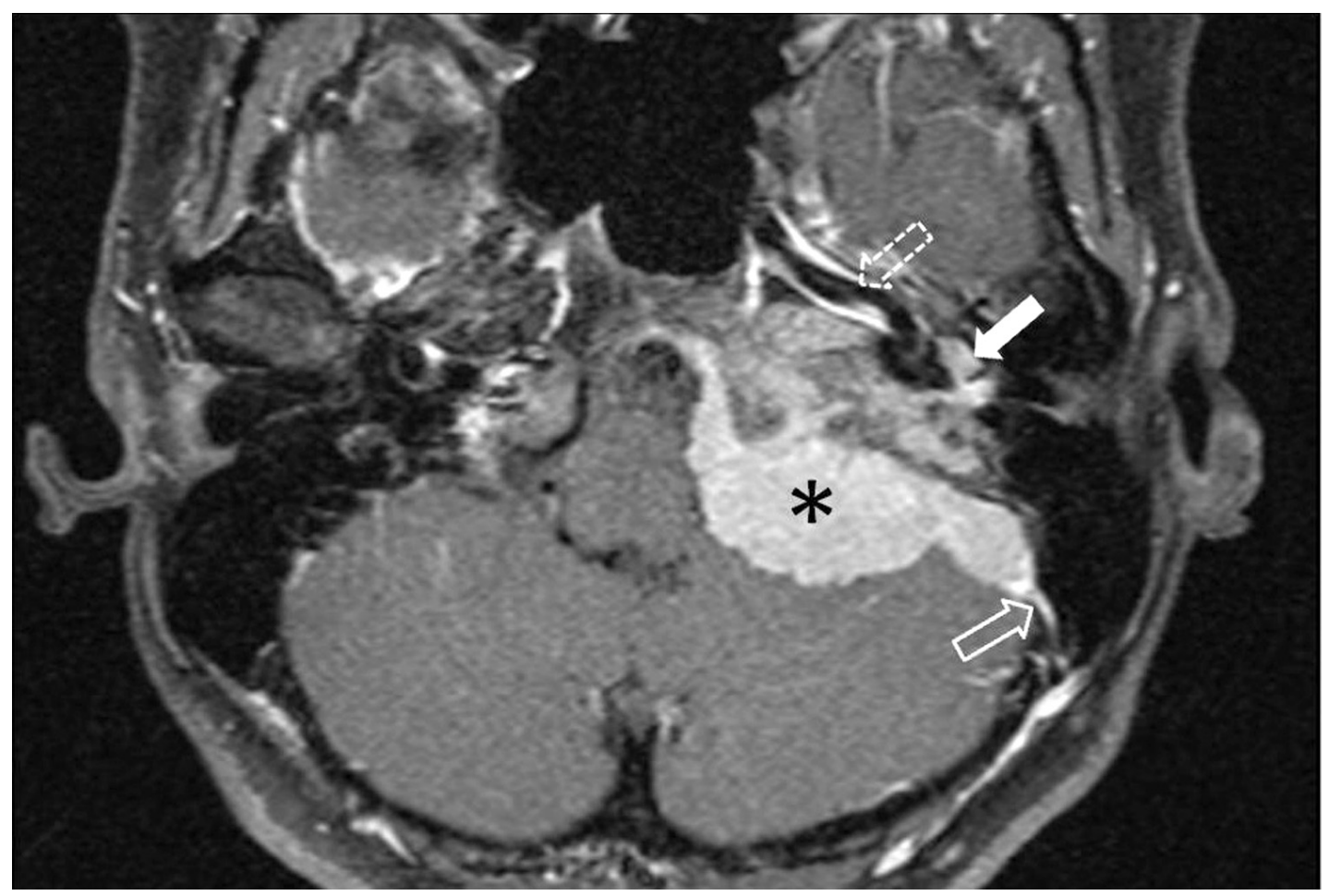

Figure 4. MRI of the Posterior Fossa, Gd-enhanced T1-weighted image, axial view, at the level of the horizontal petrous carotid artery (broken-line white arrow) showing an enhancing lesion in the left cerebellopontine angle (black asterisk) that appears to have a broad-based attachment to the posterior face of the temporal bone. There is patchy infiltration into the temporal bone with extension into the middle ear space (solid white arrow). A dural tail (white outlined arrow) is evident.

The common presenting symptoms of temporal meningioma include otalgia, hearing loss (conductive, mixed or sensorineural), ${ }^{5}$ tinnitus and facial palsy. Our patient presented with progressive unilateral hearing loss with tinnitus without history of trauma or infection. Given that otitis media with effusion is a more commonly encountered condition, it is not surprising that she was initially managed as such. When excessive bleeding was noted during myringotomy, it is not again surprising that a glomus tumor of the tympanicum type was considered, as it is the most common middle ear tumor that would present as such.

During the initial work up, the audiogram showed normal hearing in the right, and moderate to severe mixed hearing loss in the left. On review, we noted certain irregularities in the audiogram such as the inconsistent use of masking and the placement of notations for both the masked and unmasked results. The latter finding indicated a seeming hesitancy on the part of the audiologist to commit to a definite hearing level. Anecdotal clinical experience of the senior author with audiometric testing in the Philippines has shown that inaccurate audiometric examinations are not uncommon. As such, it is important to review audiometric test results with a critical eye, and to always corroborate it with tuning fork testing. In this case, tuning fork testing indicated a hearing loss with a very significant sensorineural component at $0.5,1$ and $2 \mathrm{kHz}$, a finding that was not consistent with the audiometric test results.

Repeat audiometry showed normal hearing in the right and profound hearing loss in the left. Although this finding was now consistent with tuning fork testing, it unfortunately raised suspicion regarding the true nature of the middle ear pathology. For patients with purely middle ear glomus tumors, the expected audiometric finding is a conductive hearing loss as obstruction from the mass deters conduction of sound through the middle ear. With the seemingly limited extent of the soft tissue lesion within the middle ear and jugular foramen, the sensorineural hearing loss remained unexplained. Hence, further investigation via MRI with gadolinium was warranted to rule out a retrocochlear pathology.

The gadolinium-enhanced MRI examination of our patient showed a CPA tumor with a dural tail sign causing mass effect on the adjacent pons and left cerebellar hemisphere. The dural tail sign occurs secondary to the enhancement of the thickened dura. It is the characteristic sign 


\section{FEATURED GRAND ROUNDS}

most commonly seen adjacent to a meningioma..$^{8-9}$ However, the dural tail sign has been increasingly recognized in other intracranial tumors as well. ${ }^{9}$

As a confirmatory measure, a middle ear exploratory tympanostomy and biopsy was performed. This approach provided direct access to sample the tumor without resorting to an open approach. The final histopathology report confirmed that the CPA tumor was a meningioma extending into the middle ear space.

It may be said that the diagnosis of this case was delayed from initial presentation due to the rarity of the condition. However, it may be argued that a careful analysis of the audiometric examination in relation to the findings on tuning fork testing, a simple office procedure that is often neglected, would have revealed inconsistencies that could lead to a more detailed diagnostic pathway that may have eventually led to the correct diagnosis. As such, it cannot be overemphasized that audiometric testing is not a fail-safe method of determining hearing levels, as it is dependent on the level of skill and training of the audiologist or audiometrician performing the test. Tuning fork testing although not allowing the determination of actual hearing levels does allow one to determine whether hearing loss is conductive, sensorineural or of a mixed type. If the type of hearing loss determined on tuning fork testing does not correlate with that found on standard pure tone audiometry, then the accuracy of the audiometric test must be placed under close scrutiny. An audiogram should be repeated if there is incongruence with simple clinical testing.

A cranial MRI should always be done when a patient presents with a unilateral sensorineural hearing loss to rule out any retrocochlear pathology. With the aid of appropriate ancillary procedures, along with thoughtful clinical correlation, a directed approach to diagnosing an unusual cause of hearing loss may be achieved.
1. Berkowitz $\mathrm{O}$, lyer AK, Kano H, Talbott EO, Lunsford LD. Epidemiology and environmental risk factors associated with vestibular schwannoma. World Neurosurg. 2015 Dec. 84 (6):1674-80 DOI: 10.1016/j.wneu.2015.07.007 Pubmed PMID: 26171891.
2. Bonneville F, Sarrazin JL, Marsot-Dupuch K, Iffenecker C, Cordoliani YS, Doyon D, et al. Unusual lesion of the cerebellopontine angle: a segmental approach. Radiographics. $2001 \mathrm{Mar}-\mathrm{Apr}$ 21(2): 419-38. DOI: 10.1148/radiographics.21.2.g01mr13419 PubMed PMID: 11259705.
3. Buehrle R, Goodman WS, Wortzman G. Meningioma of the temporal bone. Can J Otolaryngol. 1972; 1: 16-20.
4. Civantos F, Ferguson LR, Hemmati M, Gruber B. Temporal meningiomas presenting as chronic otitis media. Am J Otol. 1993 Jul; 14(4): 403-406. PubMed PMID: 8238280.
5. Thompson LD, Bouffard JP, Sandberg GD, Mena H. Primary ear and temporal bone meningiomas: a clinicopathologic study of 36 cases with a review of the literature. Mod Pathol. $2003 \mathrm{Mar}$; 16(3): 236-245. DOI: 10.1097/01.MP.0000056631.15739.1B PubMed PMID: 12640104.
6. Ruchenstein MJ, Cueva RA, Morrison DH, Press G. A prospective study of ABR and MRI in screening vestibular schwannomas. Am J Otol. 1996 Mar; 17(2): 317-20. PubMed PMID: 8723969
7. Isaacson JE, Vora NM, Milton S. Differential diagnosis and treatment of hearing loss. Am Fam Physician, 2003 Sep;68(6):1125-1132. PubMed PMID: 14524400.
8. WallaceEW.The dural tail sign.Radiology.2004Oct;233(1):56-7.DOI:10.1148/radiol.2331021332. PubMed PMID: 15454617.
9. Sotoudeh H, Yazdi HR. A Review on the dural tail sign. World J Radiol. 2010 May; 188-192. DOI: 10.4329/WJR.V2.15.188 PubMed PMID: 21161034 PubMed Central PMCID: PMC2999017.

